\title{
Politische Vertretung für Managed Care
}

\section{Margot Enz Kuhn, Jörg Fritschi ${ }^{b}$}

a Dr. med., Berufsverband Haus- und Kinderärzte Schweiz

b Dr. med., Präsident Schweizer Dachverband der Ärztenetze, medswiss.net

\section{* Ärztinnen und Ärzte des Managed Care-Workshops Scuol 2009 \\ Dr. med. Karsten Beer, \\ Xundart; Dr. med. Roman Buff, eastcare und Verband Managed Care Ostschweiz (vmco); Dr. med. Wolfgang Czerwenka, argomed Ärzte AG; Dr. med. Margot Enz Kuhn, Berufsverband Haus- und Kinderärzte Schweiz, argomed Ärzte AG; Dr. med. Rainer Hurni, zmed; Dr. med. Urs Keller, PizolCare; Dr. med. Alfred Rösli, HawaDoc; Dr. med. Jörg Fritschi, medswiss.net und LuMed AG; Dr. med. Felix Huber, mediX Zürich; Dr. med. Gerhard Schilling, Berufs- verband Haus- und Kinderärzte Schweiz, HAV Schaffhausen; Dr. med. Max von Salis, hapmed}

Korrespondenz: Dr. med. Jörg Fritschi Kleinbuholz 3 CH-6012 Obernau Tel. 0413201055 Fax 0413201175 j.fritischi@hin.ch
Am Managed Care-Workshop vom 10. bis 12. Dezember 2009 in Scuol haben die unten aufgeführten Mitglieder* des Berufsverbandes der Haus- und Kinderärztinnen Schweiz, «Hausärzte Schweiz» und des Schweizer Dachverbandes der Ärztenetze, «medswiss.net», beschlossen, ihre Zusammenarbeit zu intensivieren.

«Hausärzte Schweiz» und «medswiss.net» setzen sich ein für eine koordinierte, bedarfsgerechte, qualitativ hochstehende und kostenoptimierte Gesundheitsversorgung (Managed Care). Voraussetzung dafür ist eine flächendeckende, allen zugängliche Grundversorgung durch genügend und gut ausgebildete Hausärztinnen und Hausärzte. Aus diesem Grund messen beide Verbände der Förderung der Hausarztmedizin höchste Priorität bei.
- Der hausärztliche Nachwuchs ist dringend zu fördern.

- Die Hausärztin hat insbesondere in der integrierten Versorgung (Managed Care) eine zentrale Funktion.

- Managed Care wird als qualitativ hochwertiges Versorgungsmodell verstanden und soll nicht für weitere Zwecke instrumentalisiert werden.

\section{Vorgehen}

- Gemeinsame Grundsätze und Ziele und entsprechende Lösungswege werden laufend besprochen.

- Die beiden Verbände pflegen zu diesem Zweck einen festgelegten, regelmässigen Informationsaustausch (z.B. Protokolle), orientieren sich zum

\section{Wir setzen uns ein für eine koordinierte, bedarfsgerechte, qualitativ hochstehende und kostenoptimierte Gesundheitsversorgung (Managed Care)}

Aus dieser Haltung ergeben sich gemeinsame Ziele und Grundsätze. Übereinstimmende Positionen sollen erarbeitet und gezielt kommuniziert werden. $\mathrm{Zu}$ diesem Zweck treten beide Verbände, wenn immer möglich und sinnvoll, gemeinsam unter den eigenen Namen auf. Mit diesem Vorgehen erwarten deren Vertreter eine bessere Wahrnehmung und Wahrung ihrer Interessen in den gesundheitspolitischen Prozessen.

\section{Gemeinsame Grundsätze und Ziele}

- Bekenntnis für den Primat der hausärztlichen Grundversorgung als Grundpfeiler der Gesundheitsversorgung.

- Die Besserstellung der ärztlichen Grundversorger ist ein prioritäres Anliegen beider Verbände. Die hausärztliche Tätigkeit muss attraktiv bleiben und braucht gute Rahmenbedingungen.
Voraus gegenseitig über Projekte und Aktivitäten und sprechen die Zuständigkeiten, die Inhalte und das weitere Vorgehen miteinander ab.

- Bilaterale persönliche Kontakte sollen gefördert und gepflegt werden. Voraussetzung dafür ist eine gegenseitige Vertrauensbasis.

- Für die ärztliche Grundversorgung ist in erster Linie «Hausärzte Schweiz», für Managed Care «medswiss.net» zuständig.

- Divergente Positionen werden separat vertreten.

Beide Verbände sind grundsätzlich an einer Zusammenarbeit mit allen Trägern der Managed Care-Szene interessiert, insbesondere mit der FMH und dem Forum Managed Care. 